\title{
The Oblique Split Method
}

\section{A Novel Technique for Carving Costal Cartilage Grafts}

Eren Taştan, MD; Ömer Taşkın Yücel, MD; Emine Aydın, MD; Filiz Aydoğan, MD; Kaan Beriat, MD; Mustafa Gürhan Ulusoy, MD

Importance: Autogenous rib cartilage is widely used in the septorhinoplasty cases with major structural grafting requirements. However, there is a risk of warping over time.

Objective: To introduce a novel method for carving costal cartilage grafts to obtain straight grafts of varying thicknesses and to eliminate the risk of warping.

Design: Between 2007 and 2011, a total of 43 consecutive patients underwent septorhinoplasty using autogenous costal cartilage grafts carved by the oblique split method (OSM).

Setting: The Ankara Training and Research Referral Hospital, Ankara, Turkey.

Participants: The study included 43 patients with saddle nose deformity and revisional rhinoplasty with a depleted source. All patients were followed-up for a period ranging from 12 to 37 months (mean, 19.2 months) after surgery.

Interventions: All patients underwent open or closed septorhinoplasty. Autogenous costal cartilage was carved with the OSM to obtain grafts suitable for use as columellar strut, dorsal onlay, L-strut, lateral crural strut, caudal extension, and tip or speader grafts in selected cases.
Main Outcome Measures: Patients were evaluated by inspection, palpation, and photographic documentation before surgery. Inspection, palpation, and photographic documentation were carried out every 6 months and 12 months after surgery and once a year thereafter.

Resulfs: Patient satisfaction in terms of form and function was achieved in 41 patients (95\%). Two patients required reoperation for further tip projection $(n=1)$ and alar batten graft displacement $(\mathrm{n}=1)$. No complication was observed as a result of graft warping, resorption, or fracture.

Conclusions and Relevance: The OSM provides straight costal cartilage grafts of varying thicknesses without the risk of warping. Because they strictly preserve their straight form, the grafts may safely be modified into rectangular shape or carved asymmetrically and/or have their edges beveled. Current data from this study suggest that the OSM offers a flexible and reliable reconstructive option for the rhinoplasty surgeon.

\section{Level of Evidence: 4 .}

JAMA Facial Plast Surg. 2013;15(3):198-203.

Published online February 14, 2013.

doi:10.1001/jamafacial.2013.671
Author Affiliations: Departments of Otorhinolaryngology (Drs Taştan, Aydın, and Aydoğan) and Plastic, Reconstructive, and Aesthetic Surgery (Dr Ulusoy), Ministry of Health Ankara Training and Research Hospital, and Departments of

Otorhinolaryngology, Faculty of Medicine, Hacettepe University (Dr Yücel), and Faculty of Medicine, Ufuk University (Dr Beriat), Ankara, Turkey.

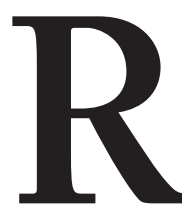

ECONSTRUCTION OF THE NAsal framework is the foundation of augmentation rhinoplasty. Autogenous cartilage grafts are preferred for this purpose because they do not induce an immune response; therefore, graft rejection, infection, and extrusion are rare. ${ }^{1}$ Autogenous cartilage graft donor sites are septal, conchal, and rib cartilage. When neither septal nor auricular cartilage is available or suitable for augmentation purposes, rib cartilage may become the only option. ${ }^{2}$ The rib offers an abundant supply of cartilage for all types of aesthetic and functional requirements and provides reliable structural support. ${ }^{3} \mathrm{Un}$ - fortunately, the costal cartilage graft has the potential to warp, which may cause distortion of the nasal form after surgery. Gibson and Davis ${ }^{4}$ reported that bending occurs because of surface tension forces and described the principle of balanced crosssectional carving to minimize warping. Gibson $^{5}$ also mentioned that the only definitive method to avoid cartilage warping is to avoid cartilage carving. There are numerous methods to minimize warping of the costal cartilage graft, particularly when it is used as dorsal onlay graft. ${ }^{4,6,7}$ Traditionally, grafts are carved along the longitudinal axis, which is perhaps the most straightforward way to harvest rectangular-profiled grafts from a round rib. 

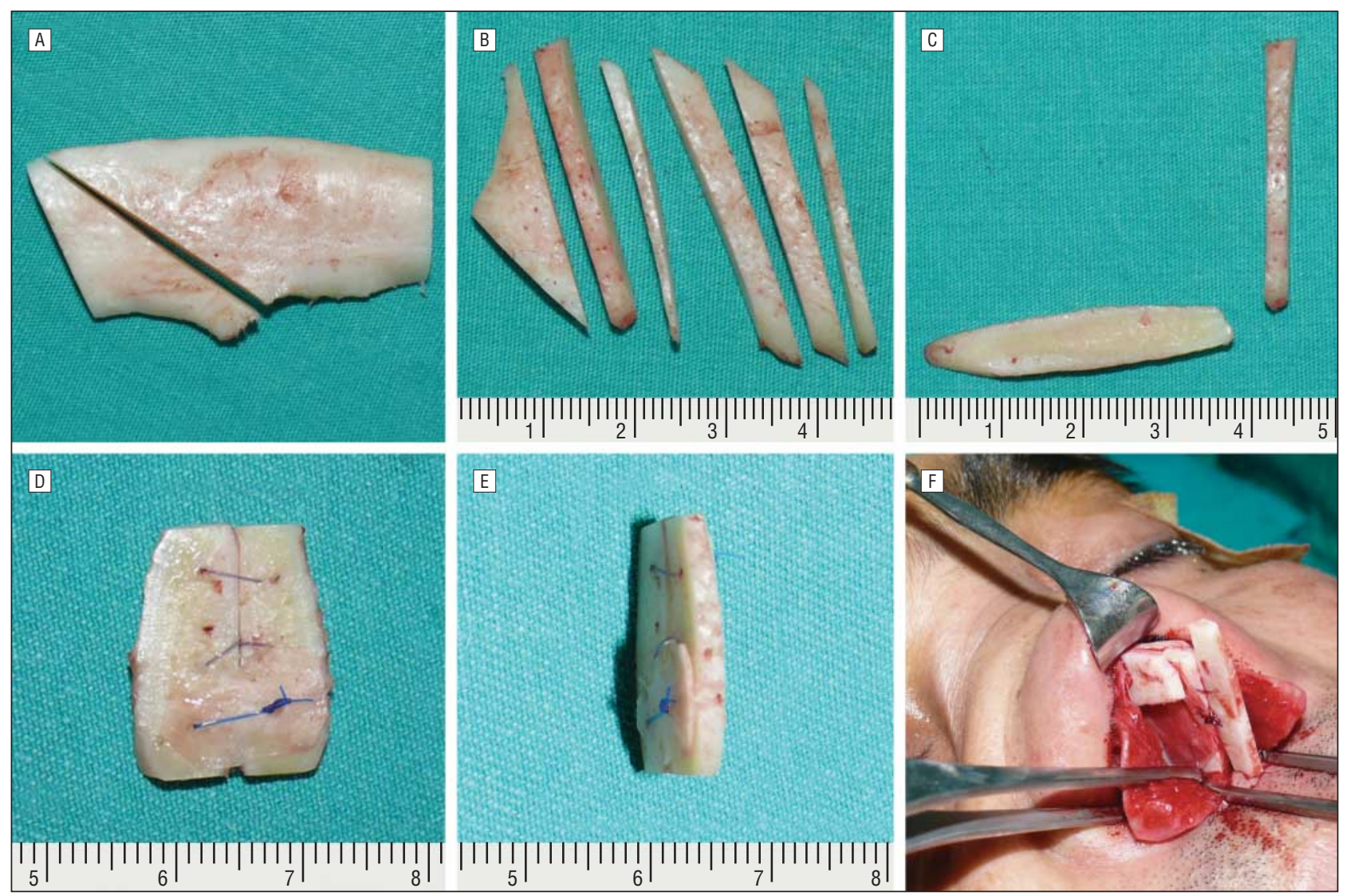

Figure 1. Splitting the seventh-rib cartilage segment and carving the grafts with the oblique split method. A, $A 45^{\circ}$ oblique split to the long axis of the rib segment. $B$ and C, Straight grafts, approximately $35 \mathrm{~mm}$ in length, with an intact surface layer surrounding the cartilage. D and E, Two cartilage segments are sewn side by side to form the caudal septal strut. A thin piece of costal cartilage graft is used for rigid fixation of the 2 segments. F, The L-strut reconstruction of septum and columellar strut. Spreader grafts reconstruct the dorsal component, and a wide caudal septal strut replaces the vertical component. A perpendicular plate of ethmoid bone graft is used for rigid fixation of the caudal strut. A columellar strut is sutured to the caudal septum. The units of measure are centimeters.

It has been suggested that centrally carved grafts exhibit a decreased rate of warping compared with peripherally carved grafts. Still, the risk of warping could not be totally eliminated with the present methods.

Herein, we describe a novel technique for costal cartilage graft carving. A cross-sectional graft obtained through an oblique cut to the long axis of the rib will result in a graft with equal circumferential forces of contracture that have a decreased chance of warping.

\section{METHODS}

The study was approved (No. 3709) by the Committee for Education, Planning, and Coordination, Ankara Training and Research Hospital, Ankara, Turkey. All patients gave informed consent to participate in the study. Between 2007 and 2011, a total of 43 patients ( 27 men and 16 women) between the ages of 19 and 55 years (mean age, 33 years) underwent primary $(n=5)$ or secondary $(\mathrm{n}=38)$ rhinoplasty with autogenous costal cartilage grafts carved by the oblique split method (OSM). An endonasal $(n=9)$ or an open $(n=34)$ approach was used with the patient under general anesthesia.

\section{SURGICAL TECHNIQUE}

A relatively straight segment of cartilaginous rib graft is harvested from the seventh rib. Usually, 3 to $4 \mathrm{~cm}$ of rib segment is enough to provide all of the grafts that are required for the nasal reconstruction. Dorsal skin softtissue and septal mucosal flaps are elevated in standard fashion. Remnants of nasal septum are exposed, and upper lateral cartilages are dissected from the nasal septum. In the presence of a dorsal and/or a caudal septal cartilage strip, a septal framework may be built over this remnant or simply reinforced by cartilage grafts. When there is inadequate septum, total septal reconstruction should be considered. After the required nasal grafts and the length of the longest monounit cartilage graft (caudal or dorsal strut graft) are determined, the rib segment is split with a high-profile microtome blade in an oblique fashion. Usually, a $45^{\circ}$ oblique split to the long axis of the rib segment will produce the requisite grafts. Also, longer grafts can be achieved by splitting the rib segment in a more oblique fashion (Figure 1 A). The OSM provides straight grafts of varying thicknesses (Figure 1B and C). In the first few patients, the carved grafts were immersed in isotonic sodium chloride solution at room temperature, kept for 1 hour, and examined for warping. Because none of the grafts showed warping during the intraoperative and postoperative periods in these patients, we discontinued waiting for early warping during the rest of the study.

Segmental reconstruction is needed only for dorsal onlay and septal replacement grafts. The L-strut reconstruc- 


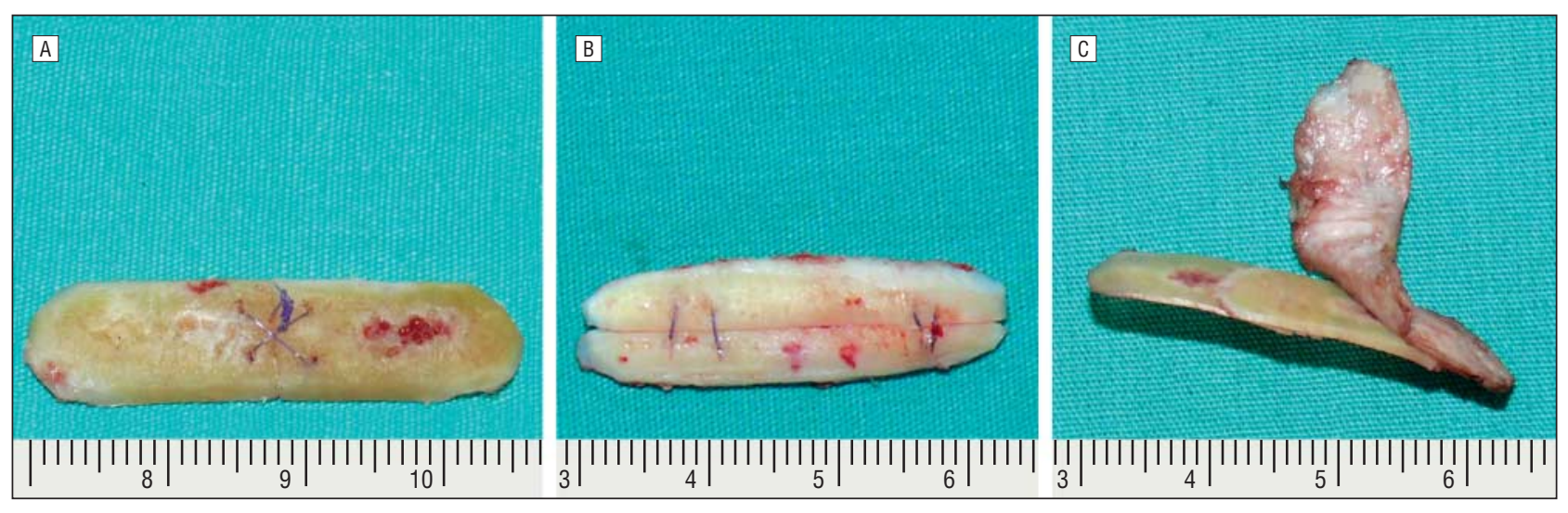

Figure 2. Dorsal onlay grafts can be obtained by 2 cartilage grafts that are sutured end to end (A), side by side (B), or by overlapping (C). To create a smooth transition, rib perichondrium may be sutured over the dorsal onlay graft. The units of measure are centimeters.

tion of the septum is the basis of successful rhinoplasty. Spreader grafts are used to reconstruct the dorsal component of the L-strut, and a caudal septal strut replaces the vertical component. A wide caudal septal strut graft can easily be prepared using 2 cartilage segments that are sewn together. A thin piece of costal cartilage graft or perpendicular plate of ethmoid bone can be used for rigid fixation of 2 cartilages, if required (Figure $1 \mathrm{D}$ and $\mathrm{E}$ ). The spreader grafts are held in proper position with 22gauge needles and fixed cephalically with a $4 / 0$ polydioxanone suture to a cartilage septal remnant or previously prepared drill holes through the caudal part of the nasal bone. Then, the caudal septal strut is placed in the proper position, and a tongue-in-groove overlap of spreader grafts on either side of the strut is achieved. Spreader grafts are kept just long enough to support the upper lateral cartilages medially. After the caudal septal strut is placed in the desired position and rotation, the dorsal height of the septum is determined by shifting the dorsal strut anteriorly and is held in place with 22gauge needles. Caudal and dorsal strut suture fixation is achieved with a $4 / 0$ polydioxanone suture. Once the Lshaped reconstruction is completed, the caudal strut is sutured to the anterior nasal spine (Figure 1F).

A dorsal onlay graft in the required width (approximately $8 \mathrm{~mm}$ in women and $10 \mathrm{~mm}$ in men) and length (average, 3.5-4.0 cm) is usually obtained with 2 segments of cartilage grafts. To obtain a dorsal onlay graft of sufficient length and width, 2 cartilage grafts can be sutured end to end, side by side, or by overlapping (Figure 2). To create a smooth transition to surrounding structures, the edges of the grafts are beveled and contoured without the risk of warping, because the forces effecting the graft surface are in equilibrium. Perichondrium harvested from the adjacent ribs can be sutured over the dorsal onlay graft to camouflage the transition between the grafts (Figure 2C). Two-point suture fixation of the dorsal onlay graft can be achieved with a $4 / 0$ polydioxanone suture attached to the underlying cartilaginous nasal skeleton. Redundant cartilage segments may be banked between the septal mucoperichondrial flaps, as these straight and thin grafts will provide additional support to the septum, without the risk of airway obstruction.

\section{RESULTS}

In this preliminary study, the follow-up period ranged from 12 to 37 months (mean, 19.2 months) after surgery. The clinical evaluation was made by inspection, palpation, and photographic documentation every 6 months, 12 months after surgery, and once a year thereafter. No complications in terms of infection, graft warping, fracture, or resorption were noted during the postoperative follow-up period. Patient satisfaction in terms of form and function was achieved in 41 patients (95\%)

(Figure 3 and Figure 4). Two patients required reoperation for further tip projection and alar batten graft displacement. These problems were caused not by distortion or resorption of the OSM grafts but by inadequate surgical planning. Both the graft banked between the septal mucosal flaps and the alar batten graft did not show any warping 6 and 10 months after surgery (Figure 5). These grafts were reused during the revision procedures without the need for any further graft harvesting.

\section{COMMENT}

Costal cartilage grafts were generally used as dorsal onlay grafts; however, in recent years, their indications have expanded vastly in rhinoplasty. In modern concept, costal cartilage grafts are used not only used as filling material but also for structural grafting. The major disadvantage of costal cartilage grafting in rhinoplasty is its tendency to warp after surgery. ${ }^{3}$

Gibson and Davis ${ }^{4}$ reported that the warping of the cartilage is caused by a difference in tension between the outermost layers of cartilage and the inner zone. An intact rib segment has these forces nicely balanced to preserve a stable shape. The matrix tends to expand when cut or carved, whereas the outer stretched layer contracts; therefore, warping occurs. The "principle of the balanced cross section" has been introduced: if the distorting forces are balanced at all points along a cartilage graft, as viewed in cross section, it will not warp. It is difficult to harvest a straight rod of cartilage from a curved rib segment without violating the principles of balanced 

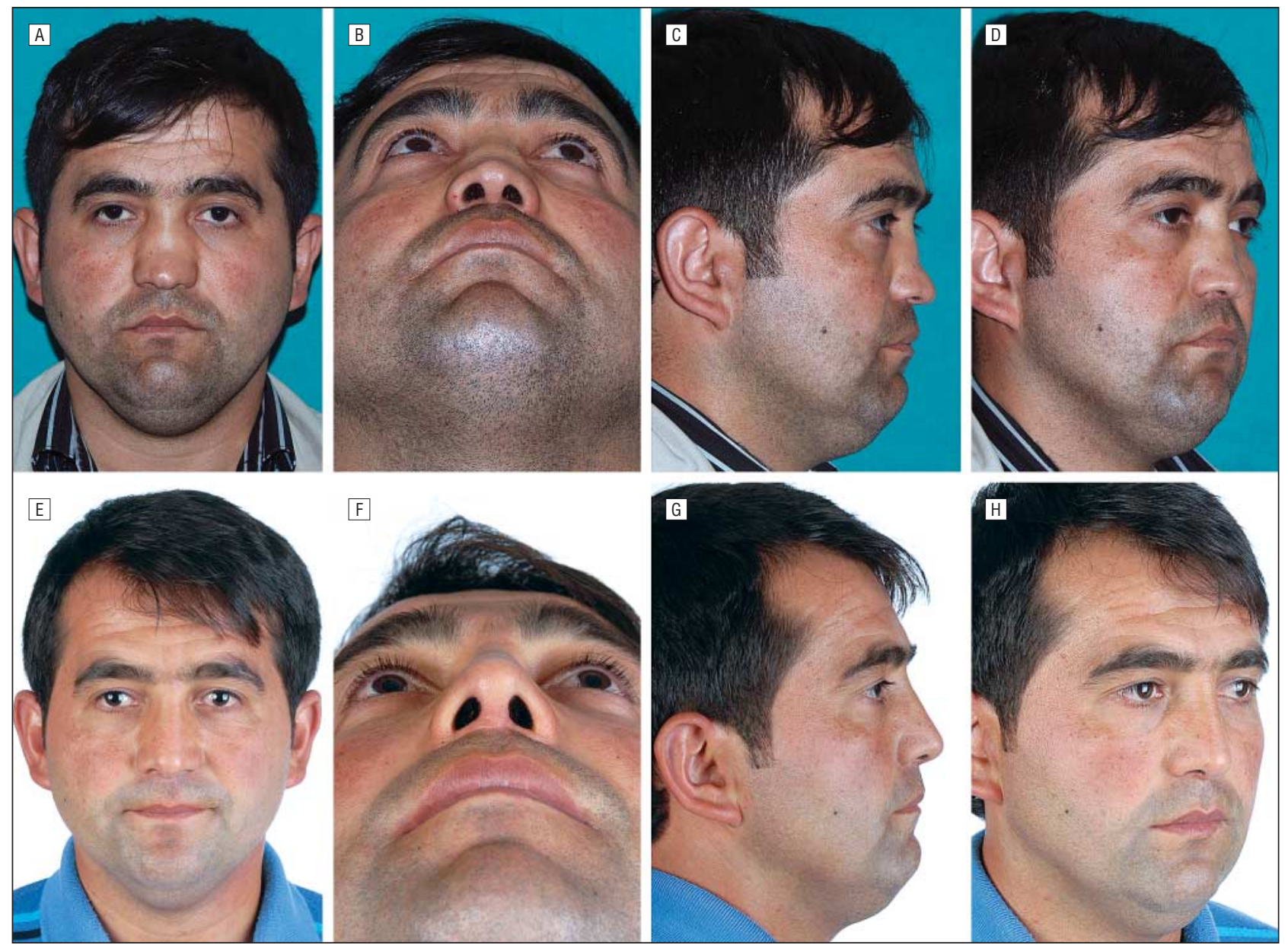

Figure 3. Preoperative (A-D) and 13th-month postoperative (E-H) views of a 28-year-old man with traumatic saddle nose deformity. Reconstruction of the L-strut was followed by placement of spreader, columellar strut, right lateral crural onlay graft, and side-by-side sutured dorsal onlay oblique split method grafts.

cross section. It is technically difficult even if the rib segment is straight, because once the outermost layer has been sliced off, the surface layer can no longer be differentiated from the deeper zones and orientation is lost. Lopez et $\mathrm{al}^{8}$ concluded that less warping occurs in centrally cut cartilage grafts than in peripherally cut grafts. They also found that the centrally cut pieces, which had a larger cross-sectional area, were less prone to warp. Gunter et $\mathrm{al}^{6}$ reported that internal stabilization of the rib cartilage grafts with Kirschner wire decreased the incidence of warping. However, extrusion of Kirschner wire, infection, pain, and numbness of the anterior palatal mucosa were observed.

The OSM provides a complete rib segment with an intact surface layer and can easily be carved according to the principle of the balanced cross section. The OSM graft's flat surface is the cross section of the rib in which the diametrically opposed forces are equal at all points on the periphery of the graft and may be said to be balanced. Because the contracting forces are in balance, the elliptical OSM grafts preserve their straight configuration even after they are modified into rectangular grafts or carved asymmetrically and/or the graft edges are beveled. This method can achieve straight grafts of varying thickness without any chance of warping. However, a curved rib cannot be modified into a straight graft by con- ventional methods without ignoring the principles of the balanced cross section.

The superoinferior caliber of the rib determines the length of the graft carved by the OSM, and the anteroposterior caliber determines the width. The grafts can be lengthened by cutting the rib at narrower angles to the long axis. Cutting the graft at $90^{\circ}$ to the long axis will result in a graft length equal to the superoinferior caliber. Decreasing the slicing angle to $45^{\circ}$ will increase the graft length about 1.4 times, whereas at $30^{\circ}$, the length of the OSM graft will be doubled compared with the graft cut at $90^{\circ}$.

On the one hand, all of the frequently used grafts in rhinoplasty (spreader, columellar strut, caudal extension, alar batten, and shield and cap grafts, to name a few) can be obtained from a single OSM graft. On the other hand, segmental reconstruction is usually required for dorsal onlay and septal replacement grafts. Segmental reconstruction of the nasal skeleton has provided consistent results. ${ }^{9}$ Segmental reconstruction of the L-strut, composed of dorsal and caudal struts, enables fine adjustment of height of the reconstructed septum. To obtain a long dorsal onlay graft that is $4 \mathrm{~cm}$ in length, 2 cartilage grafts can be sutured end to end or by overlapping after the edges are adjusted, as required. ${ }^{10,11}$ Costal perichondrium 

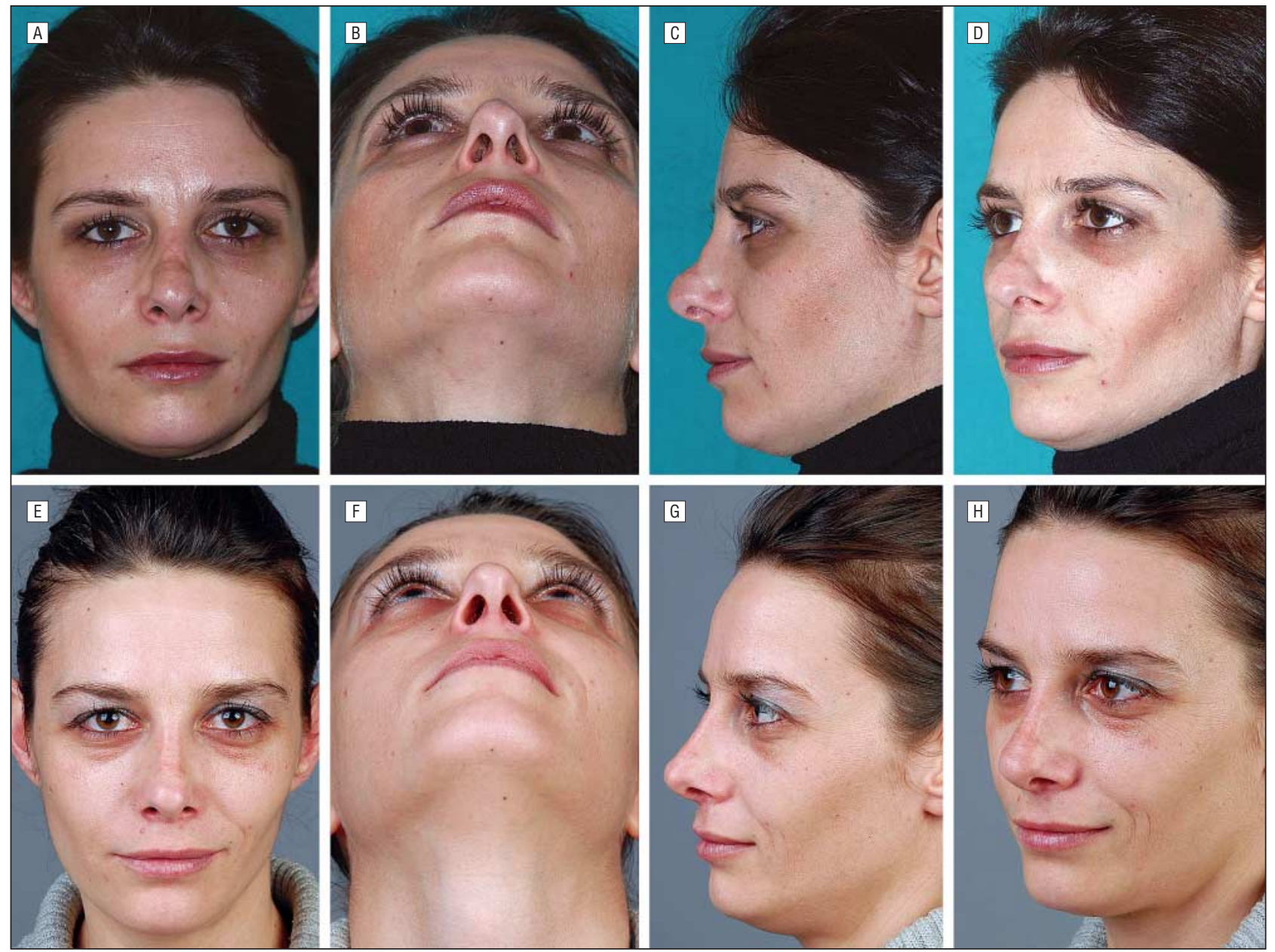

Figure 4. Preoperative $(A-D)$ and 15 th-month postoperative $(E-H)$ views of a 27 -year-old woman who had previously undergone rhinoplasty at an outside institution. Spreader, alar batten, and overlapping dorsal onlay oblique split method grafts grafts were placed through a closed approach. Because of the dorsal skin compromise, costal perichondrium was sutured over the dorsal graft for camouflage and soft-tissue padding.

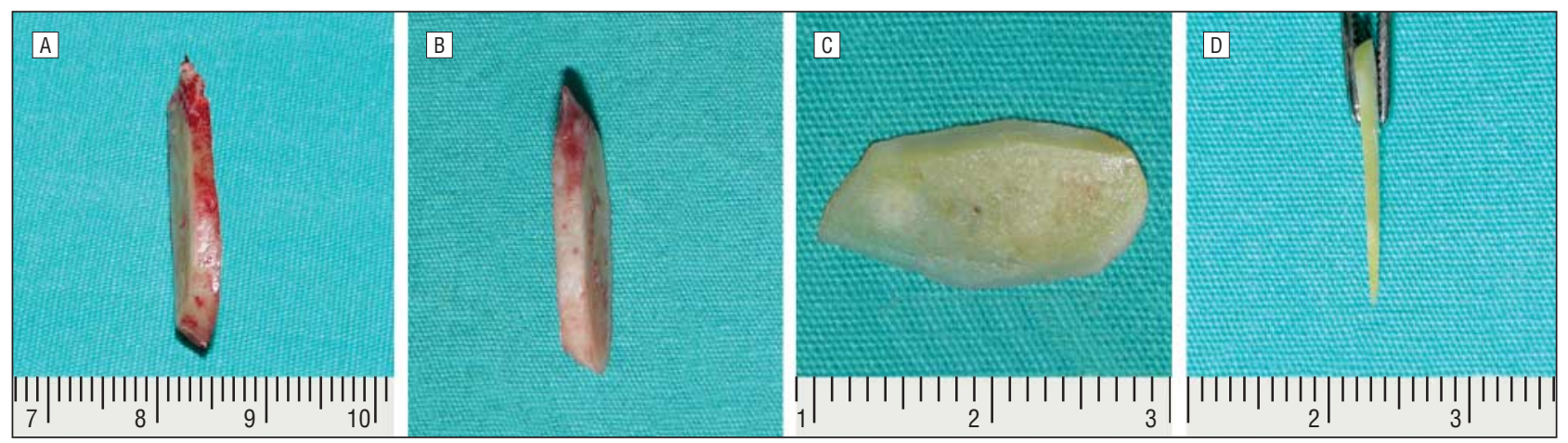

Figure 5. Intraoperative view of the oblique split method graft to be banked between the septal mucoperichondrial flaps (A) and a 6-month postoperative view of the same graft during the revision procedure (B). Ten-month postoperative views of the alar batten graft in a patient who underwent a second operation for graft displacement ( $C$ and $D)$. The oblique split method graft perfectly retained its straightness, and even, sharp, beveled edges can be seen after 10 months. The units of measure are centimeters.

can be sutured over the dorsal onlay graft to camouflage the transition between the segments ${ }^{12}$ and for soft-tissue padding.

Providing thin and straight grafts in septal cartilagedepleted patients is a challenge for the surgeon. The OSM is unique in that it provides straight grafts as thin as septal cartilage. Also, with the OSM, it is possible to obtain large quantities of graft material from the same volume of costal cartilage because the graft includes both peripheral and central portions of the rib.

In conclusion, costal cartilage grafts carved with the OSM provide grafts that are at the intended thickness, even $1 \mathrm{~mm}$, and they preserve their straight form. The OSM grafts may safely be modified into a rectangular shape, carved asymmetrically, or have their edges beveled because they strictly preserve their straight form. 
Predictable long-term results can be achieved with the OSM because it provides straight grafts of varying thicknesses without the risk of warping. The OSM is a flexible operative technique for using costal cartilage grafts in difficult structural rhinoplasty cases that require numerous grafts.

Accepted for Publication: October 22, 2012.

Published Online: February 14, 2013. doi:10.1001 /jamafacial.2013.671

Correspondence: Eren Taştan, MD, Ministry of Health Ankara Training and Research Hospital, Ulucanlar Caddesi, Altindag/Ankara, Turkey 06630 (drerentastan@gmail .com).

Author Contributions: Study concept and design: Taştan, Yücel, and Beriat. Acquisition of data: Aydin, Beriat, and Ulusoy. Analysis and interpretation of data: Aydoğan and Beriat. Drafting of the manuscript: Taştan, Aydin, Aydoğan, Beriat, and Ulusoy. Critical revision of the manuscript for important intellectual content: Yücel. Statistical analysis: Beriat. Obtained funding: Beriat. Administrative, technical, and material support: Taştan, Yücel, Aydin, and Ulusoy. Study supervision: Yücel, Aydoğan, and Beriat.

Conflict of Interest Disclosures: None reported.

Previous Presentations: This study was presented in part at the 33rd Annual Conference of the European Academy of Facial Plastic Surgery; September 4, 2010; Antalya, Turkey; and the Seventh Congress of the International Federation of Facial Plastic Surgery Societies; May 10, 2012; Rome, Italy.
Additional Contributions: We thank the patients for providing permission to share their images.

\section{REFERENCES}

1. Vuyk HD, Adamson PA. Biomaterials in rhinoplasty. Clin Otolaryngol Allied Sci. 1998;23(3):209-217.

2. Parker Porter J. Grafts in rhinoplasty: alloplastic vs. autogenous. Arch Otolaryngol Head Neck Surg. 2000;126(4):558-561.

3. Gunter JP, Cochran CS, Marin VP. Dorsal augmentation with autogenous rib cartilage. Semin Plast Surg. 2008;22(2):74-89.

4. Gibson T, Davis WB. The distortion of autogenous cartilage grafts: Its cause and prevention. Br J Plast Surg. 1958;10:257-273.

5. Gibson T. Transplantation of cartilage. In: Converse JM, ed. Reconstructive Plastic Surgery. Philadelphia, PA: WB Saunders Co; 1977:301-311.

6. Gunter JP, Clark CP, Friedman RM. Internal stabilization of autogenous rib cartilage grafts in rhinoplasty: a barrier to cartilage warping. Plast Reconstr Surg. 1997;100(1):161-169.

7. Swanepoel PF, Fysh R. Laminated dorsal beam graft to eliminate postoperative twisting complications. Arch Facial Plast Surg. 2007;9(4):285-289.

8. Lopez MA, Shah AR, Westine JG, O'Grady K, Toriumi DM. Analysis of the physical properties of costal cartilage in a porcine model. Arch Facial Plast Surg. 2007; 9(1):35-39.

9. Neu BR. Segmental bone and cartilage reconstruction of major nasal dorsal defects. Plast Reconstr Surg. 2000;106(1):160-170.

10. McKinney P. An aesthetic dorsum: the CATS graft. Otolaryngol Clin North Am. 1999:32(4):727-740.

11. Toriumi DM. Autogenous grafts are worth the extra time. Arch Otolaryngol Head Neck Surg. 2000;126(4):562-564.

12. Yotsuyanagi T, Mikami M, Yamauchi M, Higuma Y, Urushidate S, Ezoe K. A new technique for harvesting costal cartilage with minimum sacrifice at the donor site. J Plast Reconstr Aesthet Surg. 2006;59(4):352-359. 